\title{
Frequency Determined Homomorphic Unsharp Masking Algorithm on Knee MR Images
}

\author{
Edoardo Ardizzone, Roberto Pirrone, and Orazio Gambino \\ Universitá degli Studi di Palermo, \\ Computer Science and Artificial Intelligence Laboratory, \\ viale delle Scienze, Building 6, 3rd floor, Palermo, Italy
}

\begin{abstract}
A very important artifact corrupting Magnetic Resonance (MR) Images is the RF inhomogeneity, also called Bias artifact. The visual effect produced by this kind of artifact is an illumination variation which afflicts this kind of medical images. In literature a lot of works oriented to the suppression of this artifact can be found. The approaches based on homomorphic filtering offer an easy way to perform bias correction but none of them can automatically determine the cut-off frequency. In this work we present a measure based on information theory in order to find the frequency mentioned above and this technique is applied to MR images of the knee which are hardly bias corrupted.
\end{abstract}

\section{Introduction}

The RF inhomogeneity, also called Bias artifact, has been studied, in the latest years, in a lot of works related to this kind of artifact which afflict medical images and in particular Magnetic Resonance Images (MRI). The magnetic field of the device has intensity variations that depend on the scan sequence, the patient tissues and the kind of coil used. In particular, surface coil image is more sensible to this artifact but noise is not strong; on the contrary, body coil image has the opposite behavior. As a consequence of this phenomenon, the image is corrupted by a very low frequency signal whose visual effect is a non uniform illumination which can be seen either as white stripe, or as a more or less extended peak of luminance or an image shading along a direction where the magnetic field force lines go away. This is especially true if those images are produced by open coil devices like the one used for lower and upper limbs imaging. The approaches used to suppress this artifact can be subdivided in two classes: discovering the degradation model or modifying classic segmentation algorithms to classify the tissues taking into account the degradation model. In the first class of methods, a phantom,usually a container holding a known substance, may be introduced in the device with the aim to obtain an image afflicted by magnetic field intensity variations without any interaction with tissues and discover the degradation model. With this special experimental setup, each slice is corrected thanks to this special image that can be considered like a surface which approximates the illumination variations [7] 10. If body-coil image and surface-coil image of the 
same slice are available, a preconditioned gradient algorithm can be used to discover the surface[2] after the input images have been registered. In [1] the bias artifact is modelled by Legendre polynomials and the algorithm estimates each uncorrupted class computing the image by a least-square technique, and assuming that it is made by piecewise zones with constant intensity level. The above methods can be considered evolutions of homomorphic filtering approaches 16 . moving from [8] where the HUM (Homomorphic Unsharp Masking) has been developed. This approach either uses directly the phantom pixel to perform the correction or performing a log subtraction of the image with a blurred version of the same one using low pass filters[11]. In the last years has been developed the second class of methods oriented to modify classical segmentation algorithms to take into account, in their iterative steps, the artifact effect. This first approaches used the EM algorithm[12, 6] [4 while some more recent works deal with modified fuzzy c-means (fcm) algorithm[3] 14, 15] are used for their higher speed with respect to the previous one. An approach exclusively based on information theory is proposed in [13] which provides an unusual solution to the problem, minimizing the entropy of the inverse degradation model with Brent-Powell algorithm. The question is open because the NEUROVIA PROJECT [17] shows that this artifact isn't uncorrelated with tissues. This project was born as comparative study of six different algorithms with the aim to determine, thanks to a large and accurate experimental setup in various environment conditions, the best approach on encephalic MR: the result was that each algorithm shows a different bias estimation, due to its theoretical approach, and by the interaction of the tissues with the magnetic field. We perform our experimental setup on images of knee which are not shown in any study presented above and are corrupted by heavy bias artifact. The rest of the paper is arranged as follow. Sections 2 and 3 describe the proposed method for bias removal. In section 4 the experimental setup is detailed, while section 5 reports some conclusions.

\section{The Adopted Filtering Scheme}

Often in medical imaging the image is composed by a background and a foreground so if an homomorphic filtering is performed a streak artifact is produced on the boundary. Guillemaud [5] proposed an homomorphic filtering where the Region of Interest (ROI) is considered to avoid the artifact cited above. The algorithm is explained into detail as follow:

1. define a binary image (background $=0$; foreground $=1$ ) in order to define the region of interest named $R O I$

2. perform a log-transform to the original image with background suppressed:

$$
I_{\log }=\log (I(R O I))
$$

3. apply a low-pass butterworth filter of 2nd order in frequency domain obtained from the previous step:

$$
I_{\text {filt }}=F F T^{-1}\left(\text { Butterworth } h_{\text {order }=2}\left(F F T\left(I_{\text {log }}\right)\right)\right)
$$


4. perform the same butterworth filtering to the binary image:

$$
R O I_{\text {filt }}=F F T^{-1}\left(\text { Butterworth } h_{\text {order }=2}(F F T(R O I))\right)
$$

5. due to anti-transform operation, the images will be complex data, so perform magnitude of each pixel value:

$$
I_{\text {mag }}=A B S\left(I_{\text {filt }}\right) \quad R O I_{\text {mag }}=A B S\left(R O I_{\text {filt }}\right)
$$

6. divide pixel-by-pixel the ROI pixels of the image obtained at the step 3 with that one at step 4 :

$$
\log (\text { Bias })=I_{m a g} / R O I_{m a g}
$$

7. subtract the image at step 2 with that one obtained at the previous step and perform an exponential transform:

$$
I_{\text {restored }}=\exp \left(I_{\text {log }}-\log (\text { Bias })\right)
$$

8. perform a contrast stretching of the image at step 7 in order to obtain the initial dynamic:

$$
I_{\text {corrected }}=\frac{I_{\text {restored }}-\min \left(I_{\text {restored }}\right)}{\max \left(I_{\text {restored }}\right)-\min \left(I_{\text {restored }}\right)} \cdot \max (I)
$$

The Bias image can be obtained in this way:

$$
I_{\text {Bias }}=\exp (\log (\text { Bias }))
$$

and performing the same stretching adopted in (1). An unresolved problem of this method is that the butterworth filter cutoff frequency (bfcf) is not specified: this is an important parameter because the entity of homomorphic process depends by this one. If it is too low, no effect will be visible on $I_{\text {corrected }}$; on the contrary, higher values of bfcf will produce on $I_{\text {corrected }}$ a loss of tissues contrast, that is tends to uniform gray level. For values of bcfc close to 1, produce a total inversion between $I_{\text {corrected }}$ and $I_{\text {Bias }}$ : $I_{\text {Bias }}$ will appear identical to the initial image $I$ while $I_{\text {corrected }}$ is made by a quasi-uniform gray level in the ROI, as shown in fig.1.
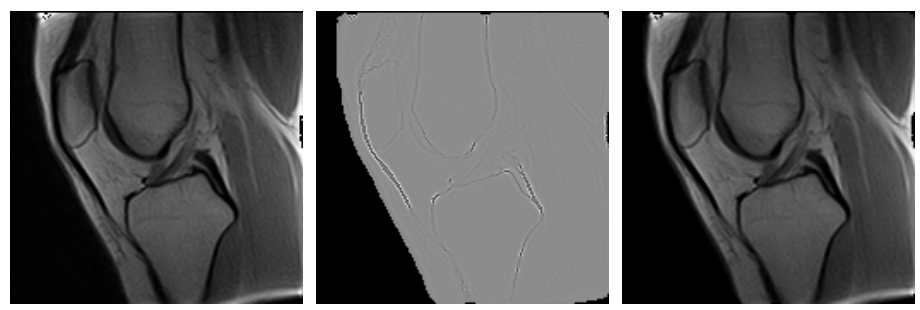

Fig. 1. Cut-off frequency 0.99 - From Left to Right: original, filtered and bias images

This fact is a classic image processing finding because step 4 corresponds to an high-pass filtering so the lower frequencies which are subtracted from $I$ are given to $I_{\text {Bias }}$. 

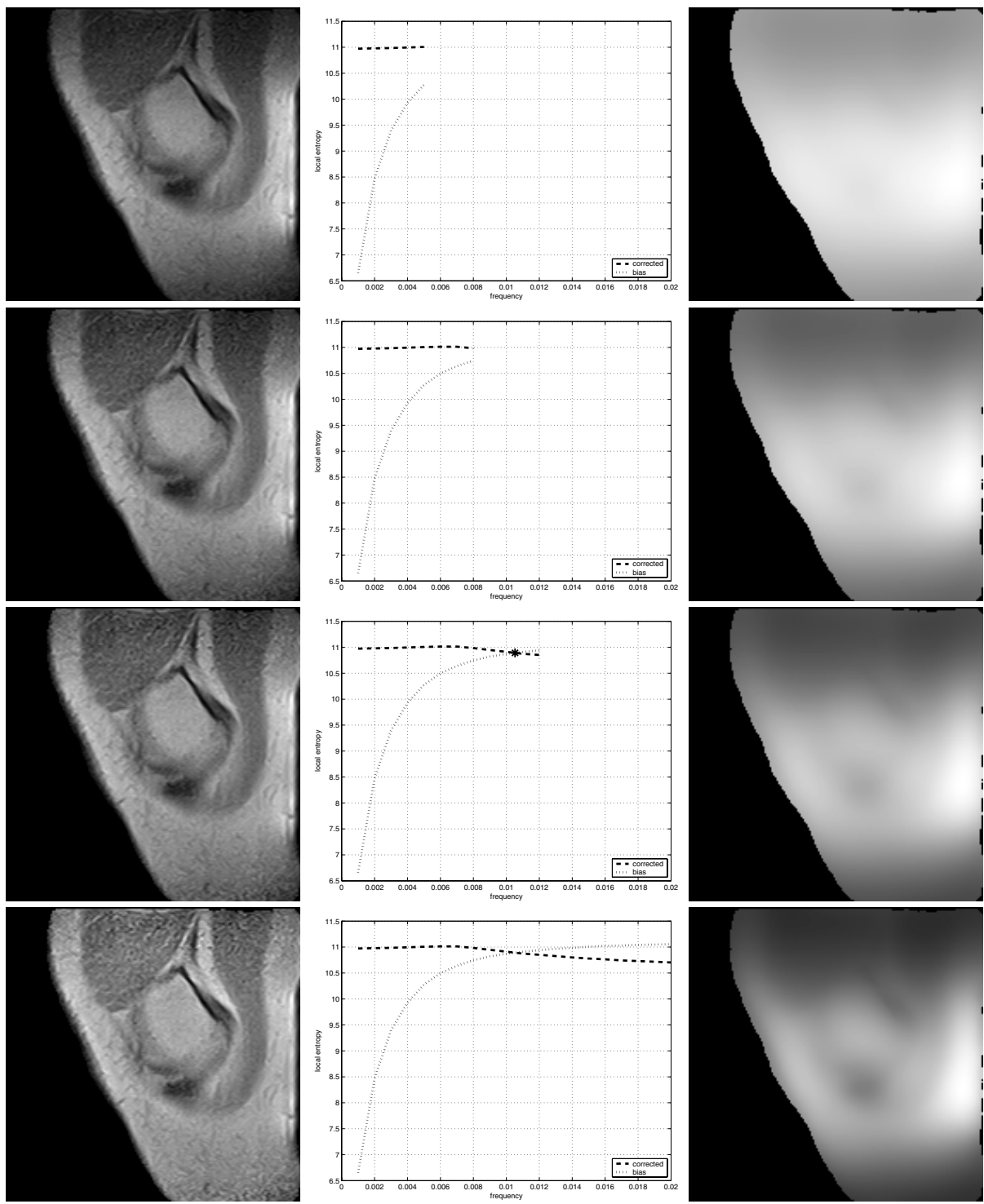

Fig. 2. Up-to-down: sequences at growing cut-off frequency. From Left-to-Right: corrected, diagram frequency-entropy,bias estimated.

\section{Cut-Off Frequency Determination}

From the information theory point of view, $I$ has more information than necessary: it contains both not corrupted information and bias information, so the filtering can be considered like a sort of information transfer. It is necessary, then, introduce a function which measure the quantity of information which 
is contained in an image: in correspondence of the ROI, it could be seen, according to a growing cut-off frequency,an increment of the information in $I_{\text {Bias }}$, while in $I_{\text {corrected }}$ should happen an information decreasing. The Shannon Entropy is a such information measure which has been already used in literature [6] 13:

$$
H=-\sum p(x) \cdot \log _{2}[p(x)]
$$

where $x$ is the image histogram. In fig. 2 this information transfer is shown. As it can be noticed, the corrected image has been chosen in correspondence of the intersection point of the two curves which are the local entropies both $I_{\text {corrected }}$ and $I_{\text {Bias. }}$. The entropy is calculated only on ROI, because the background doesn't contain any information about the artifact.

\section{Experimental Setup}

The method has been performed on images decoded from DICOM file format, that is without any optical scanner acquisition which may introduce other artifacts. The device is an ESAOTE ARTOSCAN C with a magnetic field intensity of 0.18 Tesla . The dataset which we present in this paper is a complete study of the knee on sagittal plane which consists of $19 \mathrm{~T} 1$-weighted images acquired with the following parameters: Spin Echo sequence, Repetition time: $980 \mathrm{~ms}$, Echo time: $26 \mathrm{~ms}$, Slice thickness: 4mm, Flip Angle= 90. The useful resolution of FOV is $170 \times 170$ pixels with 12 bit of pixel depth. Both the original and restored images have been submitted to two physicians specialized in orthopaedy for a visual inspection. Only two restored images exhibit a gray level distortion, but they maintain the contrast among the tissues. All the restored images shows a panoramic illumination showing details on peripheral zone of the images, while in the original ones only the central zone of the anatomy is over illuminated. After the subjective evaluation performed above, the coefficient of variation has been performed on each zone showed in fig. 3

$$
c v(z o n e)=\frac{\sigma(z o n e)}{\mu(z o n e)}
$$

where $\sigma$ and $\mu$ are, respectively, standard deviation and mean of the zone. As it can be seen in fig. 4 , the $c v$ decrease in almost all the zones.


Fig. 3. Form left to right:original image, restored image, manual segmentation, table of coefficient of variations 

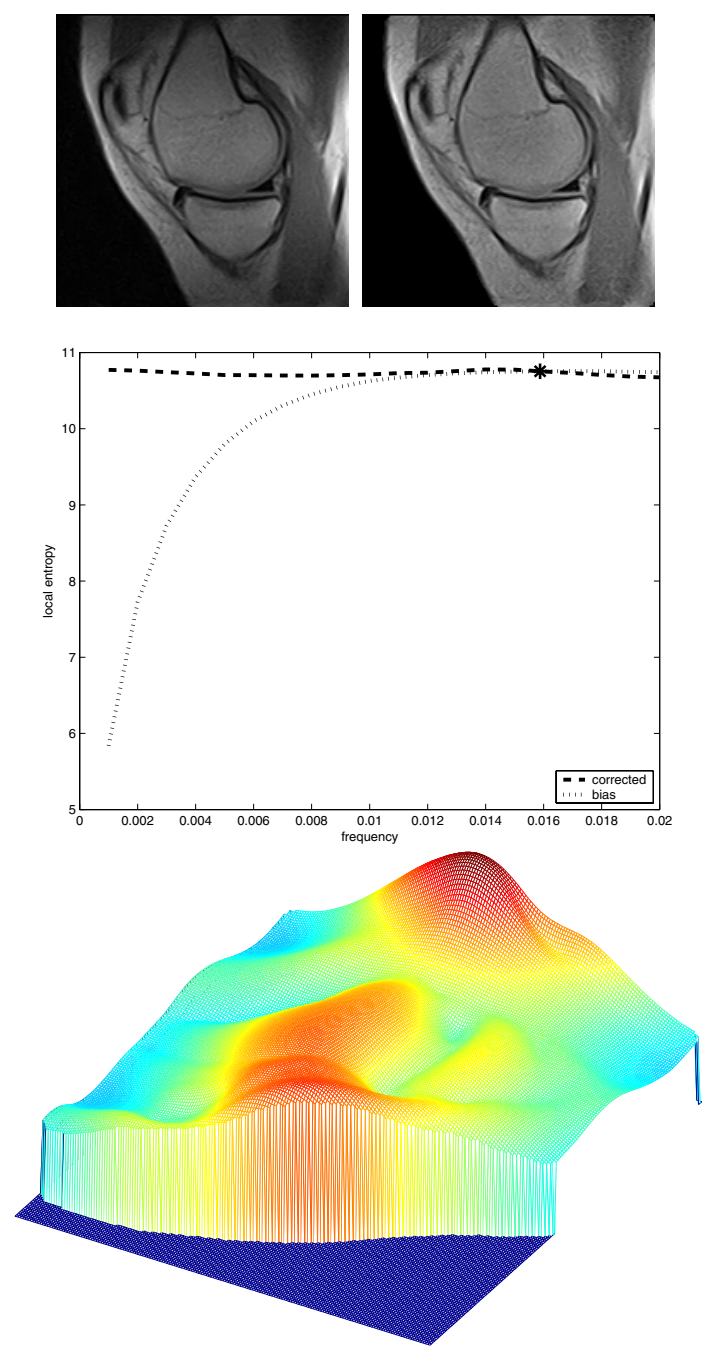

Fig. 4. Up to Down:original and restored images; local entropy diagram; Bias surface

\section{Conclusions}

An automatic technique has been presented to determine the cut-off frequency in homomorphic filtering for bias removal in MRI. The presented method exhibit several good properties:

- no hypothesis are made on tissue classes. It can be applied on wide kind of medical images; often the application presented in other papers is focused usually on encephalic MRI.

- Implementation is quite simple: it is an improvement of classical filtering approach in image analysis. 
- There is no needs of preliminary experimental setup to "tune" the filter.

- The method performs on the fly and it is fully adaptive and automatic.

The method can be implemented in semi-automatic way in order to allow the doctor to set a preferred setting, so the cut-off frequency is only suggested by the algorithm. This is not a marginal fact: the doctor uses visual inspection of the image with the aim of to determine the disease.

\section{Acknowledgement}

This work has been partially supported by Istituto Radiologico PIETRO CIGNOLINI - Policlinico dell'Universitá di Palermo. Particulars thanks Dr. Daniele Peri for his technical support, Dr. Gian Piero De Luca and Dr. Claudio Cusumano for their medical support and prof. Giuseppe De Maria for his availability.

\section{References}

1. Styner, M.; Brechbuhler, C.; Szckely, G.; Gerig, G.: Parametric estimate of intensity inhomogeneities applied to MRI Medical Imaging. IEEE Transactions on Medical Imaging 22 (2000)153-165

2. Shang-Hong Laia; Ming Fangb: A dual image approach for bias field correction in magnetic resonance imaging. Magnetic Resonance Imaging 21 (2003)121-125

3. Mohamed N. Ahmed; Sameh M. Yamany; Nevin Mohamed: A Modified Fuzzy CMeans Algorithm for Bias Field Estimation and Segmentation of MRI Data. IEEE Transactions on Medical Imaging 21 (2002) 193-199

4. Van Leemput K.;Maes F.; Vandermeulen D. and Suetens P.: Automated ModelBased Bias Field Correction of MR Images of the Brain. IEEE Transactions on Medical Imaging 18 (1999) 885-896

5. Guillemaud, R.: Uniformity Correction with Homomorphic filtering on Region of Interest. IEEE International Conference on Image Processing 2 (1998) 872-875

6. Guillemaud, R.; Brady M. : Estimating the Bias Field of MR Image. IEEE International Conference on Image Processing 2 (1998) 872-875

7. Dawant B.M.; Zijdenbos A.P.; Margolin R.A.: Correction of Intensity Variations in MR Images for Computer-Aided Tissue Classification. IEEE Transactions on Medical Imaging 12 (1993) 770-781

8. Axel L.; Costantini J.; Listerud J.: Intensity Correction in Surface Coil MR Imaging. American Journal on Roentgenology 148 (1987) 418-420

9. Lei Jiang,Wenhui Yang:A Modified Fuzzy C-Means Algorithm for Segmentation of Magnetic Resonance Images. Proc. VIIth Digital Image Computing: Techniques and Applications.Sun C., Talbot H., Ourselin S. and Adriaansen T. Editions. (2003) $225-231$

10. Tincher M.; Meyer C.R.; Gupta R.; Williams D.M.: Polynomial Modelling and Reduction of RF Body Coil Spatial Inhomogeneity in MRI. IEEE Transactions on Medical Imaging 12 (1993) 361-365

11. Brinkmann B. H. , Manduca A. and Robb R. A.: Optimized Homomorphic Unsharp Masking for MR Grayscale Inhomogeneity Correction. IEEE Transactions on Medical Imaging. 17 (1998) 161-171 
12. Wells W.M.; Grimson W.E.L.; Kikins R.; Jolez F.A.: Adaptive Segmentation of MRI Data. IEEE Transactions on Medical Imaging. 15 429-442 (1996)

13. Likar B.; Viergever M.A.; Pernus F.: Retrospective Correction of MR Intensity Inhomogeneity by Information Minimization. IEEE Transactions on Medical Imaging 20 (2001) 1398-1410

14. Pham D.L.; Prince J.L.: Adaptive Fuzzy Segmentation of Magnetic Resonance Images. IEEE Transactions on Medical Imaging. 18(9), (1999) 737-752

15. Pham D.L.; Prince J.L.: An Adaptive Fuzzy C-Means Algorithm for Image Segmentation in the Presence of Intensity Inhomogeneities. Pattern Recognition Letters. 20(1), (1999) 57-68

16. Gonzalez R.C.; Woods R.E.: Digital Image Processing. Prentice Hall Ed.

17. Arnold JB; Liow J-S; Schaper KS; Stern JJ; Sled JG; Shattuck DW; Worth AJ; Cohen MS; Leahy RM; Mazziotta JC; Rottenberg DA. Quantitative and Qualitive Evaluation of Six Algorithms for Correcting Intensity Non-Uniformity Effects. Neuroimage (2001) 13(5) 931-943. 